\title{
La importancia de la imagen país: El caso de los Estados Unidos
}

\author{
Gina Pipoli de Azambuja \\ Ph.D. ( c ) en Economía y Dirección de Empresas por la Universidad de Deusto, España; \\ Magíster en Administración con especialización en Marketing, Licenciada en Administración de Empresas \\ y Contadora Pública Colegiada de Universidad del Pacífico. \\ Profesora e Investigadora del Departamento Académico de Administración de la \\ Universidad del Pacífico, Lima, Perú.
}

\section{Resumen}

La imagen país es un tema que está cobrando cada vez mayor importancia en los últimos años, debido a la competencia que se está generando entre los países por captar turistas e incrementar sus exportaciones a raíz de la globalización. Actualmente, los países se preocupan más por la imagen país que están proyectando y, por consiguiente, de la percepción favorable o desfavorable que los consumidores tendrán del país.

La presente investigación constituye un estudio exploratorio cuyo objetivo es determinar los componentes de la imagen país de los Estados Unidos, desde la perspectiva de los consumidores del Perú y de Francia. Se eligió a los Estados Unidos para efectos del presente estudio porque es el país del que proviene el mayor flujo turístico al Perú y constituye el principal mercado de destino de su oferta exportable; mientras que en el caso de Francia ocurre todo lo contrario: tiene un bajo índice de turistas y de comercio. Ello permite realizar la comparación de los resultados de dos muestras: una con un alto nivel de familiaridad (Perú) y otra con un bajo nivel de familiaridad (Francia), dado que en ambos mercados se aplicó el mismo cuestionario, bajo las mismas condiciones, y se midieron las mismas variables e indicadores.

Palabras clave: Imagen país (Country image), identidad nacional (Country identity), Efecto país de origen (Country of origin effect).

\section{Introducción}

A lo largo de las últimas décadas, el número de empresas que busca expandir sus operaciones y tener presencia comercial más allá de sus fronteras se ha incrementado sustancialmente. Lo mismo ha sucedido con los países cuyo objetivo es generar ventajas competitivas en el mercado global en el que compiten por introducir sus exportaciones.
Por ello, los países compiten por ganar presencia en los mercados internacionales, ya sea mediante un incremento de su oferta exportable o de la atracción de mayor cantidad de turistas o inversiones (Kotler, P. y D. Gertner 2002).

De este modo, la estrategia de marca país, una de las estrategias del marketing de lugares que en los últimos años está adquiriendo mayor reconocimien- 
to. Se ha convertido en una herramienta fundamental para poder construir y modelar la imagen de los países, de tal forma que puedan alcanzar un posicionamiento consistente y efectivo.

Considerando este contexto que impone la necesidad de aplicar una visión de marca país y dado el fenómeno de la globalización, la imagen país es un tema muy investigado desde los años 1970, cuando Nagashima (1970: P. 68) define la imagen país como la percepción de los consumidores relativas a las ideas, bagaje emocional y connotación asociada con un concepto. Es así que el «hecho en» se refiere a las imágenes, reputación, y estereotipos que los consumidores atribuyen a los productos elaborados en un país en particular. El siguiente hito importante se da cuando Han y Terpstra (1988) operacionalizan este constructo analizando las catorce variables del estudio de Nagashima. Los estudios en torno a este tema han continuado hasta la fecha, aportando a la literatura las consideraciones por tener en cuenta en la estrategia de marketing internacional de los países. Sin embargo, la tarea de construir una imagen país sólida y favorable es quizá el mayor reto que cualquier nación desearía superar, pues al mismo tiempo se deben considerar los elementos que construyen esta imagen de la nación; la identidad nacional y el efecto país de origen.

Los Estados Unidos constituyen un referente de gran importancia en el tema de marca país, no solo por tratarse de una gran potencia mundial sino por el importante sitial que ha logrado mantener en el ranking del Country Brand Index (CBI) de Futurebrand ${ }^{1}$ : siempre ha logrado ocupar uno de los tres primeros lugares y en el año 2009, su marca país se ubicó en el primer lugar en el mundo. Por ello, los demás países de la región vuelcan su mirada en la forma cómo se ha manejado la marca país Estados Unidos, con el fin de determinar cuáles son las lecciones por aprender para lograr su desempeño.
En la primera parte del documento se presenta una revisión de la literatura sobre la imagen país y las variables que la conforman; es decir, la identidad nacional y el efecto país de origen. En la segunda parte, se presentan los resultados de la parte empírica: los componentes que conforman la imagen país de los Estados Unidos según la percepción de los consumidores del Perú y Francia. Dado que esta investigación es de carácter exploratorio, sus resultados se consideran como preliminares para futuras investigaciones en torno a este tema.

\section{Objetivos de la investigación}

El objetivo general de la investigación fue determinar cuáles son los factores que influyen en la percepción del público con relación a la imagen del país.

Los objetivos específicos de la investigación fueron:

- Conocer la percepción de la imagen país de los Estados Unidos entre los peruanos y franceses.

- Determinar los principales componentes de la imagen país de los Estados Unidos para los peruanos y franceses.

\section{Revisión de la literatura}

\section{La identidad nacional}

La identidad puede definirse como la esencia de algo $o$ alguien; mientras que la imagen se refiere a cómo ese algo es percibido, lo cual ciertamente refleja un carácter bastante subjetivo. La brecha existente entre ambos conceptos -identidad e imagen- puede representar un factor negativo, pues en muchas ocasiones los países son percibidos por lo que no son en realidad. Esto último demuestra una clara distorsión entre la identidad y la imagen, lo que permite verificar el

1. Disponible en: <http://www.futurebrand.com/cbi/overview/>. 
carácter subjetivo atribuido a la imagen ( $0^{\prime}$ Shaughnessy y Jackson 2000; Kotler y Gertner 2002).

El desarrollo de una imagen país favorable debe comenzar por la construcción de una identidad nacional, lo cual es un proceso complejo porque se deben identificar qué elementos, de los múltiples que integra la identidad nacional, resultan importantes y favorables para los objetivos planteados en la campaña de desarrollo de la estrategia de marca-país (Dinnie 2008).

Entre los varios componentes que definen la identidad de una nación destacan: la geografía del país, así como su historia, sus manifestaciones culturales, su música, sus ciudadanos reconocidos y la iconografía expresada mediante símbolos patrios, como la bandera nacional (Smith 1991; Tuck 2003). En relación con la cultura, Burguess y Harris (1999) resalta que uno de los temas más sensibles respecto de la identidad nacional es la evidente tensión entre la diversidad cultural y la unidad nacional, dado que la identidad de la marca país, que constituye la herramienta mediante la cual se construirá su imagen, debe considerar a todo el país. Una marca país no es propiedad de un gerente de marca ni de los líderes de la nación; por el contrario, le pertenece a todo el país, a cada uno de sus ciudadanos (Kotler et al. 1993).

\section{El efecto país de origen}

El país de origen en los últimos años ha adquirido mayor relevancia frente al contexto mundial por la expansión de las políticas de libre mercado, que facilitan el intercambio comercial a gran escala. Es una variable que ha sido ampliamente estudiada a través de los años por diversos investigadores. (Samiee 1994) define el país de origen como el país con el que una firma es asociada, el cual normalmente es el país de origen de la firma ${ }^{2}$. Schooler (1965) fue el primero en identificar las predisposiciones a los productos, sobre la base de su país de origen. Desde entonces, el efecto país de origen ha sido identificado en una gran cantidad de estudios.

Por otro lado, existen estudios que sostienen que el efecto país de origen no existe o que tiene un marginal impacto (Johansson et al. 1985; Samiee 1987; Olson y Jacoby (1972) y Erickson et. al (1984). Años más tarde, otros autores coincidieron en definir el efecto país de origen como el impacto que tiene el país de origen de cierto producto o servicio en la percepción, actitudes, evaluación y comportamiento del consumidor ante esta oferta (Cordell 1991; Chao 1993; Peterson y Jolibert 1995).

Sin embargo, la mayoría de estudios apunta a que el efecto país de origen existe, a pesar de que no se termina de definir aún su magnitud y el mecanismo cómo funciona. En este sentido, la investigación desarrollada por Bilkey y Ness (1982) consignó como objetivo inicial de la investigación, la validación de la tesis que señalaba que el efecto país de origen influía significativamente en la evaluación global que hace el consumidor ante una determinada oferta. Sin embargo, los resultados obtenidos demostraron que el efecto país de origen es un atributo entre los muchos que caracterizan a un producto. Se debe reconocer que un producto posee atributos intrínsecos (como tamaño, color y calidad) y atributos extrínsecos (el precio, por ejemplo) y, por lo tanto, en ausencia de otras señales de información, el consumidor podrá utilizar el efecto país de origen para evaluar el producto. Por ello, recomendaron que este factor no fuera tomado como la única variable del modelo (Bilkey y Nes 1982). Asimismo, se ha demostrado que la influencia del efecto país de origen en la evaluación de los consumidores es más fuerte cuando los consumidores no estaban familiarizados con una categoría de producto, y utilizaban al efecto país de origen como una variable que facilitaba la evaluación en la ausencia de otros criterios en Erickson, Johansson y Chao 1984)).

2. Traducción del autor. 
Es importante mencionar que recién a partir de 1994 se empieza a cuestionar la relevancia del país de origen, en especial en lo referente a su incidencia en el comportamiento de compra (Samiee 1994). Años más tarde, investigadores como Usunier (2006) cuestionan aun más la relevancia de esta variable del producto, al señalar que aspectos como la globalización de la producción y las marcas globales ocasionaron que el factor relativo al país de origen perdiera relevancia.

El carácter del efecto país de origen que generan los estereotipos de cada nación puede ser universal. Sin embargo, el grado en que este influye en la evaluación de los productos por parte del consumidor, varía en función del país (Papadopoulos et al. 1987). Peterson y Jolibert (1995), por su parte, afirman que el efecto se configura como un concepto dependiente que solo puede existir bajo ciertas condiciones.

Una manifestación clara del efecto país de origen se citó en la investigación llevada a cabo por Gaedeke (1973), cuando encontró que los productos de los Estados Unidos eran percibidos como de mejor calidad que aquellos fabricados en países menos desarrollados. Adicionalmente, encontró que las marcas específicas podían ser evaluadas mejor o peor, según el nivel de desarrollo económico del país de origen, para luego utilizar la marca como información de comparación. Otro factor que se encontró fue que en los países desarrollados, los productos locales tendían a ser evaluados de manera más favorable que los importados (Morello 1984).

Por último, cabe señalar que Dinnie (2008) señala que la publicidad también constituye un medio relevante en la exposición del país de origen, pues mediante la imagen país puede o no establecerse una relación entre el producto o servicio publicitado y el país de origen.

\section{La imagen país}

Luego de definir los elementos que conforman la identidad de una marca país y reconocer el impac- to del efecto país de origen en la decisión de compra del consumidor, se debe analizar cómo proyectar todas estas imágenes, conceptos y asociaciones relativas a una nación al resto del mundo, para la construcción de la imagen país deseada.

En primer lugar, se debe reconocer que la imagen país representa un concepto abstracto. Sin embargo, las distintas investigaciones desarrolladas en torno a este tema, por lo general, convergen en una definición bastante similar pero con distintos matices. Kotler y Gertner (2002) descomponen el concepto y señalan que la imagen país representa un gran conjunto de asociaciones e información relacionada con una nación determinada, pero de manera simplificada, como resultado de un proceso mental, al tratar de acopiar la información más relevante sobre este lugar. Por otro lado, otros autores definen la imagen país como el conjunto total de creencias que una persona conserva de los productos de un determinado país (Nagashima, 1970; Han y Terpstra, 1988; Darling y Wood 1990).

Por su parte, Dinnie (2008) señala que los componentes que construyen la imagen país son: la experiencia personal; que implica la familiaridad con el país mediante el contacto con el mismo; las referencias recibidas; los eventos políticos; las marcas de productos y servicios de este país; la imagen de sus líderes de opinión; los estereotipos y los medios de comunicación.

Los resultados de estas definiciones de imagen país permiten establecer que la imagen país constituye una percepción simplificada de una nación. Esta percepción, en muchas circunstancias, da lugar a la creación de estereotipos que no necesariamente representan interpretaciones exactas de la realidad, pero que pueden ejercer un efecto positivo, negativo o neutral frente a la imagen país (Higson 1997).

Además de la identidad y la imagen, Kotler y Keller (2006) señalan que es necesario manejar el posicionamiento de la marca país, ya que sin ella todos los esfuerzos desplegados en el desarrollo 
de la imagen de marca no tendrían sentido. Así, el posicionamiento de cualquier marca y, aún más, de una marca país depende de dos importantes procesos previos: la diferenciación y la segmentación (Keller 2003; Bauer et al. 2006).

Finalmente, cabe señalar que de acuerdo con la revisión de la literatura, existen dos enfoques por los cuales se construye la imagen país: la micro imagen país y la macro imagen país. El primer enfoque se refiere a las asociaciones y percepciones generadas en torno a productos y/o categoría de productos de determinada nación (Agarwal y Sikri 1996); mientras que el segundo, desarrollado por Martin y Eroglu (1993) y que ha sido el adoptado para la presente investigación, define a la imagen país como el conjunto de información y creencias que una persona tiene sobre un país en particular.

En el gráfico 1 se presenta el modelo conceptual utilizado para la presente investigación, en el que se plantea que los dos grandes componentes de la imagen país son: la identidad país y el efecto país de origen. La variable identidad país se refiere a los elementos intrínsecos del país que recogen su esencia, como su geografía, historia, cultura, ciudadanos reconocidos e iconografía; mientras que la variable efecto país de origen representa los elementos extrínsecos al país relacionados con las estrategias de marketing del país, vale decir, el comportamiento del consumidor, la publicidad, las estrategias de marca y la promoción de las exportaciones.

El modelo conceptual presentado en el gráfico 1 descompone la imagen país en aquellos constructos que intervienen en su conformación.

La identidad nacional y el efecto país de origen se reflejan y proyectan por la imagen país, que se construye sobre la base de percepciones generadas por la experiencia personal y la familiaridad con el país. Es decir, aquellos recuerdos que las personas tienen del contacto directo con el país, de las referencias respecto de esa nación, de los eventos políticos, de la reputación de los líderes de opinión, del conocimiento de las marcas locales y, finalmente, de los medios de comunicación (Kotler y Gertner 2002; Dinnie,2008).

\section{Gráfico 1}

\section{Modelo conceptual de imagen país}

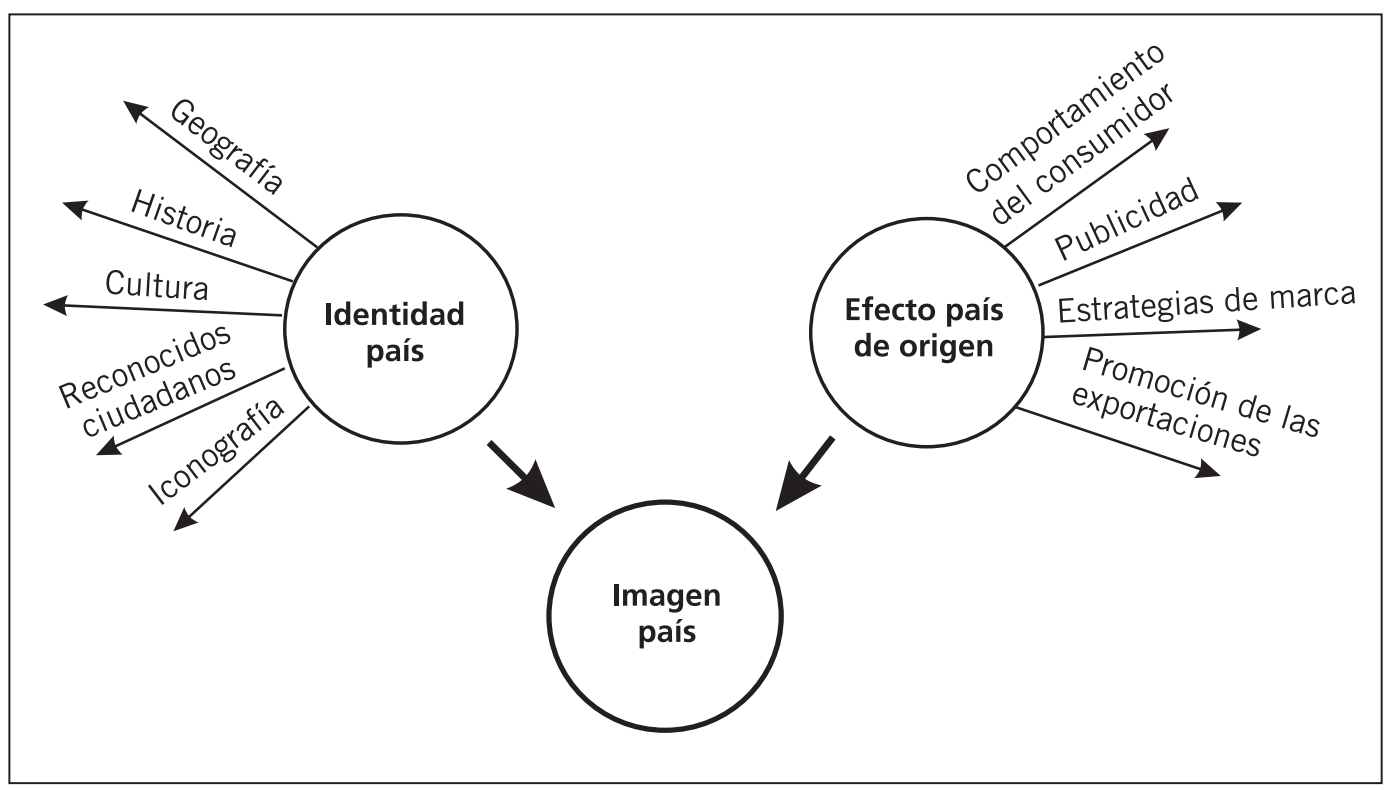




\section{Metodología}

La investigación, en su primera etapa, consistió en la revisión de la literatura existente sobre imagen país y los elementos que la conforman: la identidad nacional y el efecto país de origen, lo cual implicó la revisión de cada uno de las variables que conforman dichos constructos. En la segunda etapa de la investigación se realizó una contrastación empírica que permitiera reconocer la imagen país asociada a los Estados Unidos de América entre los pobladores del Perú y de Francia. Para ello, se analizaron las percepciones de los consumidores de dos mercados, en función de su nivel de familiaridad con esta potencia mundial, expresada mediante sus relaciones comerciales.

\section{Composición y tamaño de la muestra}

De acuerdo con la revisión de la literatura realizada sobre la imagen país y el efecto país de origen, Al-Sulaiti y Baker (1998), en la revisión de la literatura que realizaron sobre el efecto país de origen desde 1965, muestran que las investigaciones realizadas sobre este tema utilizan como sujeto de unidad muestral a los estudiantes y el tamaño de la muestra es menor a 100 en cada caso.

Al respecto, Johansson et al. señalan que utilizaron en su investigación dos muestras por conveniencia de estudiantes de postgrado (70 estudiantes de los Estados Unidos y 82, de Japón) porque, si bien no eran representativas del país en general, constituían dos muestras comparables de dichos países en lo que respecta a sus características socioeconómicas, que constituyen factores que pueden afectar sus evaluaciones.

Se trabajó con estudiantes de posgrado por considerarse que tendrían un mayor nivel educativo y cultural; y, por lo tanto, mayor exposición a los elementos que conforman la imagen proyectada por otros países. El muestreo fue del tipo no probabilístico.
En nuestro caso, la muestra estuvo conformada por estudiantes de la Maestría en Administración (MBA): 122 pertenecían a universidades en el Perú y 115 , en Francia. La restricción de edad considerada fue que fueran mayores de 18 años, de modo que fueran compradores potenciales de toda la oferta exportable de los Estados Unidos y que tuviesen la capacidad de viajar a dicho país. Se excluyó a los extranjeros, en cada uno de los países estudiados, dado que la investigación tenía interés en conocer la percepción de la imagen de los Estados Unidos entre consumidores peruanos y franceses.

En el cuadro 1 se presentan las características de las dos muestras. En él se observa que las dos muestras son comparables entre sí en términos de edad, sexo y nivel de estudios, que constituyen las variables socioeconómicas consideradas en este estudio. La mayor diferencia reside en la edad de los encuestados, aunque no es significativa.

\section{Cuadro 1}

\section{Características de la muestra}

\begin{tabular}{|lrr|}
\hline & \multicolumn{1}{c}{ Perú } & Francia \\
\hline Edad promedio & 28 años & 22 años \\
Sexo (\% masculino) & $57 \%$ & $54 \%$ \\
Estudios de posgrado & $100 \%$ & $100 \%$ \\
& & \\
Nacionalidad francesa & - & $100 \%$ \\
Número de encuestados & $100 \%$ & 122 \\
Nacionalidad peruana & 115 & $(51,5 \%)$ \\
Número de encuestados & $(48,5 \%)$ & - \\
\hline
\end{tabular}

Elaboración propia sobre la base de la investigación realizada.

Recolección de la data

La recolección de la data se realizó en agosto de 2008 a los estudiantes del Programa de Maestría en la Universidad del Pacífico. Los cuestionarios fueron presentados a cada muestra durante las horas de clase (Johansson et al. 1985). El instrumento de recolección de información utilizado fue un cuestionario estructurado de preguntas abiertas y cerradas. 
Los cuestionarios fueron elaborados en español. La versión del cuestionario para Francia fue traducida al inglés, dado que su MBA era en inglés; luego, nuevamente se tradujo del inglés al español, para asegurar que no hubiera distorsiones por el idioma (Douglas y Craig 1983). Se realizó una prueba piloto con quince consumidores de la muestra en cada uno de los países seleccionados con el fin de verificar su operatividad y, en función de los resultados, realizar los ajustes que fueran necesarios.

El cuestionario, que fue autoadministrado por los estudiantes, tuvo cuatro partes, de acuerdo con la estructura empleada por Lawrence et al. (1992):

1.- Comportamiento de compra

2.- Actitud hacia el país de origen

3.- Familiaridad

4.- Información demográfica.

Okechuku (1994) señala que la investigación realizada hasta la fecha sobre el país de origen sugiere que el origen del efecto país en evaluaciones de los productos, por parte de los consumidores, varía en función de los siguientes factores:

- El grado de disponibilidad, la familiaridad y la capacidad de servicio percibida de los productos extranjeros frente a los productos locales.

- La complejidad técnica de los productos.

- Las creencias de los consumidores acerca de la conveniencia de comprar productos fabricados en el extranjero.

- La percepción del nivel de desarrollo económico del país de origen.

- El grado de similitud de los sistemas económicos, culturales y políticos del país extranjero con el país de origen.

Asimismo, el autor menciona que aunque muchos estudios sugieren que el país de origen afecta las evaluaciones de productos de consumo y las intenciones de compra, poco se sabe sobre el efecto de la presencia de atributos de producto.
Por ello, se trabajó con dos muestras: una conformada por los consumidores del Perú y otra por los consumidores de Francia, habiéndose elegido estos dos países en función del nivel de familiaridad de sus consumidores con los Estados Unidos. De esta forma, el Perú constituía el país en el que sus ciudadanos tienen un alto nivel de familiaridad con los Estados Unidos, expresado por los acuerdos de comercio exterior y cooperación entre el Perú y dicho país. Del mismo modo, se eligió a Francia como el país en el que sus ciudadanos tienen un bajo nivel de familiaridad con los Estados Unidos porque su intercambio comercial es poco significativo (véase el cuadro 2).

\section{Cuadro 2}

\section{Distribución de las exportaciones hacia el Perú y Francia desde los Estados Unidos}

\begin{tabular}{|l|c|c|}
\hline País & $\begin{array}{c}\text { Exportaciones } \\
\text { hacia el Perú, } \\
2007 \\
\text { (En dólares) }\end{array}$ & $\begin{array}{c}\text { Exportaciones } \\
\text { hacia Francia, } \\
2007 \\
\text { (En dólares) }\end{array}$ \\
\hline Estados Unidos & 27.819 .120 & 4.119 .530 \\
\hline
\end{tabular}

Fuente: International Trade Center (www.trademap.org) Elaboración propia.

\section{Características de la investigación realizada}

La investigación realizada fue de tipo exploratorio porque no existen estudios sobre este tema realizados en el Perú y Francia. Para ello se realizó una investigación de tipo cualitativa en la que se analizó la percepción de los consumidores peruanos y franceses respecto de la imagen de los Estados Unidos.

\section{Discusión y análisis de los resultados}

Discusión sobre la imagen país de los Estados Unidos de América

Los Estados Unidos de América constituyen una nación conformada por muchos elementos que generan diversas asociaciones en torno a ella, tales como personajes famosos, guerras, tecnología, desarrollo económico, lugares turísticos, etcéte- 
ra. Sin embargo, hasta el momento no han logrado construir una imagen país única, que conjugue todos los valores de la nación en un solo concepto, que integre los elementos que constituyen su identidad nacional al mismo tiempo que los de su efecto país de origen.

A lo largo de los últimos diez años, se han puesto en marcha muchas iniciativas con miras a promover el turismo, tal es así que se han creado marcas ciudad de mucho éxito, como es el caso de Nueva York o San Francisco. Sin embargo, no se ha trabajado una clara estrategia de marca país para posicionar a los Estados Unidos con un concepto determinado en la mente de los consumidores. A pesar de ello, en las tres últimas ediciones del Country Brand Index $(\mathrm{CBI})$, los resultados fueron muy favorables para los Estados Unidos. Así, se tiene que en las versiones del CBI de Futurebrand, para los años 2006 y 2007, logró ubicarse en el puesto número dos del ranking general de marca país. En el año 2008, el país descendió al tercer lugar del ranking, para posicionarse en el primero en el año 2009.
La evolución de los resultados de la información del CBI, en el año 2007, ubicó a los Estados Unidos en el primer puesto de cinco categorías, tal como se puede observar en el cuadro 3.

Finalmente, cabe señalar que durante el año 2008, los Estados Unidos captaron la atención mundial por sus elecciones presidenciales. Si bien la figura de Barack Obama aún no se ve reflejada en la investigación de imagen país realizada, probablemente si aparecerá en los años futuros.

\section{Análisis de los resultados}

En el cuadro 4 se presentan los resultados del levantamiento de la data recolectada para la parte empírica de la investigación con relación a los componentes de imagen país Estados Unidos.

Finalmente, cabe señalar que durante el año 2008, los Estados Unidos captaron la atención mundial por sus elecciones presidenciales. Si bien la figura de Barack Obama aún no se ve reflejada en la investigación de imagen país realizada, probablemente si aparecerá en los años futuros.

\section{Cuadro 3}

Evolución de indicadores de la marca país Estados Unidos

\begin{tabular}{|c|c|c|c|c|c|c|}
\hline \multicolumn{5}{|c|}{$\begin{array}{l}\text { Los Estados Unidos han visto una notoria declinación } \\
\text { en muchos direcccionadores claves en la mentedel consumior }\end{array}$} & \multirow{2}{*}{\multicolumn{2}{|c|}{$\begin{array}{c}\text { Los Estados Unidos } \\
\text { no estan entre los top de } \\
\text { estas categorías clave }\end{array}$}} \\
\hline $\begin{array}{c}\text { Rankings } \\
2007\end{array}$ & $\begin{array}{r}\text { Ranki } \\
200\end{array}$ & & \multicolumn{2}{|c|}{ Variación 2008-2007 } & & \\
\hline$\# 1$ & $\# 2$ & Facilidad para hacer negocios & $\boldsymbol{\Delta}+6,8 \%$ & Familiaridad & \# 14 & Estandar de vida \\
\hline$\# 1$ & \# 6 & Extender viajes de negocios & $\boldsymbol{\nabla}-4,5 \%$ & Preferencia & \# 19 & Libetad política \\
\hline$\# 1$ & $\# 2$ & Conferencias & $\boldsymbol{\Delta}+3,1 \%$ & Consideración & \# 45 & Seguridad \\
\hline$\# 2$ & \# 5 & Vida nocturna & $\boldsymbol{\Delta}+9,8 \%$ & Visita & \# 62 & Medio ambiente \\
\hline \# 4 & $\# 8$ & Familias & $\boldsymbol{\nabla}-7,2 \%$ & Devoción & & \\
\hline \# 5 & $\# 8$ & Deportes y aire libre & & & & \\
\hline \# 6 & \# 7 & Mejor para vivir & & & & \\
\hline
\end{tabular}

Fuente: Country Brand Index 2008; Futurebrand

Elaboración: Futurebrand 
Análisis de los resultados

En el cuadro 4 se presentan los resultados del levantamiento de la data recolectada para la parte empírica de la investigación con relación a los componentes de imagen país Estados Unidos.

Los ciudadanos encuestados del Perú y Francia indicaron qué personaje, lugar, monumento o cualquier otro concepto asociaban con los Estados Unidos de América. Como puede apreciarse, por el abultado porcentaje de la respuesta "Otros», existe una gran dispersión de los resultados, lo cual constituye un indicador de que no existe una imagen única y clara de los Estados Unidos entre los ciudadanos encuestados del Perú (país con una alta familiaridad con dicho país) ni entre los ciudadanos franceses encuestados (baja familiaridad).

Por otra parte, puede apreciarse que los lugares y monumentos representan un conjunto importante de conceptos asociados a los Estados Unidos, entre los cuales destacan: Iugares y atractivos turísticos (Nueva York, Estatua de la Libertad, Torres Gemelas, Los Ángeles, Washington D.C. y Hollywood), con menciones de alrededor del 15\% del total de resultados. Asimismo, se observa la fuerte presencia de marcas globales asociadas a la imagen de los Estados Unidos, como es el caso de Disney y Mc Donalds, que constituyen el segundo grupo de importancia en los conceptos asociados a su imagen. En lo que respecta a personajes famosos asociados a la imagen del país, resalta la mención a George W. Bush, que era el Presidente del país al momento de la aplicación de las encuestas, por lo que su asociación con la imagen de los Estados Unidos, se hace evidente.

En el caso de Francia, los dos factores encontrados como los principales componentes de la imagen país Estados Unidos fueron: George Bush y Mc Donalds; mientras que en el caso del Perú fue un solo factor: Disney. Así, según los ciudadanos franceses encuestados, los principales componentes de la imagen de los Estados Unidos estaban referidos a un líder político famoso -como era su presidente en ese momento- $y$ a una marca de gran presencia e importancia en dicho país y en el mundo entero. Entre los ciudadanos peruanos encuestados, solo se encontró un elemento que estaba referido también a una marca famosa de los Estados Unidos: la marca Disney. Cabe destacar que ambas marcas se encuentran en el rubro diversión y entretenimiento.

\section{Cuadro 4}

\section{Componentes de la imagen país Estados Unidos}

\begin{tabular}{|l|c|c|c|c|}
\hline & Perú & Francia & Perú & Francia \\
\hline George Bush & $\%$ & $\%$ & $\begin{array}{c}\text { Número de } \\
\text { encuestas }\end{array}$ & $\begin{array}{c}\text { Número de } \\
\text { encuestas }\end{array}$ \\
Nueva York & 4,8 & 11,9 & 6 & 15 \\
Estatua de la Libertad & 4,0 & 8,7 & 5 & 11 \\
Mc Donalds & 4,0 & 5,6 & 5 & 7 \\
Disney & 2,4 & 11,9 & 3 & 15 \\
Poder & 12,9 & 0,0 & 16 & 0 \\
Hamburguesas & 1,6 & 4,8 & 2 & 6 \\
Comida rápida & 0,8 & 6,3 & 1 & 8 \\
Torres Gemelas & 4,0 & 0,8 & 5 & 1 \\
Los Angeles & 1,6 & 0,8 & 2 & 3 \\
Washington & 0,0 & 2,4 & 0 & 0 \\
Hollywood & 1,6 & 0,0 & 2 & 1 \\
Otros & 0,0 & 0,8 & 0 & 58 \\
\hline Total & 62,1 & 46,0 & 77 & 126 \\
\hline
\end{tabular}

Elaboración propia sobre la base de los resultados de la investigación realizada. 


\section{Conclusiones}

Luego de analizar los resultados correspondientes a las dos muestras analizadas, se puede observar que no existe una imagen país única de los Estados Unidos entre los encuestados. Por el contrario, esta se encuentra muy fragmentada porque no se ha trabajado una estrategia de posicionamiento clara y constante, a lo largo de los años, que permita acuñar un concepto en la mente del público. En vista de la ausencia de un esfuerzo de marketing internacional constante en el tiempo que permita fijar un determinado concepto con claridad, la imagen se ha generado por sí sola, recogiendo aquellos elementos que han tenido un mayor impacto en la percepción de los pobladores de los países analizados. En este caso, se han referido a un líder político y a marcas globales muy vinculadas con su país de origen, como son Mc Donalds y Disney.

Este problema se debe a que la estrategia de posicionamiento de los Estados Unidos no ha sido elaborada a partir de los elementos fundamentales de la identidad nacional y del efecto país de origen, como señala la literatura, sino que se ha ido formando en base a los hechos relevantes que se han ido sucediendo a través de los años, a sus ciudades más importantes que han cobrado rele- vancia a través de los años, así como en función a los personajes famosos que han destacado en el país.

Definitivamente, aún queda mucho que investigar respecto de este tema. Sin embargo, esta investigación constituye un primer paso para conocer la percepción de los consumidores peruanos y franceses que estudiaron una maestría respecto de los componentes de la imagen país de los Estados Unidos.

El punto de partida radica en mirar a los componentes fundamentales de la marca país, como son la identidad nacional, con todo el bagaje cultural del país y, por el otro lado, al efecto país de origen, que implica las percepciones de los consumidores generadas por las estrategias de marketing, los productos originarios del país, el conocimiento del país, su nivel de competitividad en el mercado internacional, entre otros. Para ello, se recomienda que en futuras investigaciones se mida el impacto de la identidad nacional y del efecto país de origen en la imagen país en un número mayor de países, con una muestra representativa del universo, de modo que sus resultados puedan ser extrapolados al universo y se pueda ver el efecto del presidente Obama en la imagen de los Estados Unidos. 


\section{Referencias}

AGARWAL, S. y S. SIKRI

1996 «Country Image: Consumer Evaluation of Product Category Extensions». En: International Marketing Review, vol. 13, No 4, pp. 23-39.

AL-SULAITI, K. L. y M. J. BAKER

1998 "Country of Origin Effects: A Literature Review». En: Marketing Intelligence \& Planning, vol. 16, №3, pp. 150-199.

\section{BAILEY, W. y S. A. GUTIERREZ DE PINERES}

1997 "Country of origin attitudes in Mexico: The malinchismo effect Journal of International Consumer Marketing; 1997; Vol. 9, No. 3; pp. 25-42. ABI/INFORM Global

BAUER, A.; B. BLOCHING y K. HOWALDT

2006 Moment of Truth: Redefining the CEO's Brand Management Agenda. Reino Unido: Palgrave Macmillan.

BILKEY, W. J. y E. NES

1982 "Country-of-Origin Effects on Product Evaluations». En: Journal of International Business Studies, vol. 13, N 1, pp.89-99

BRODOWSKY, G. H.

1998 «The Effects of Country of Origin and Country of Assembly on Evaluations about Automobiles and Attitudes toward Buying Them: A Comparison Between Low and High Ethnocentric Consumers». En: Journal of International Consumer Marketing, vol. 10, $\mathrm{N}^{\circ} 3$, pp. 85-113.

\section{BURGESS, S.M. y M. HARRIS}

1999 «Social Identity in an Emerging Consumer Market: How You Do the Wash May Say a Lot about who You Think You Are». En: Advances in Consumer Research, vol. 26, $\mathrm{N}^{\circ} 1$, pp. 170-175.

CHAO, P.

1993 "Partitioning Country of Origin Effects: Consumer Evaluations of a Hybrid Product». En: Journal of International Business Studies, vol. 24, N², pp. 291-306.
1989 "The Impact of Country Affiliation on the Credibility of Product Attribute Claims». En: Journal of Advertising Research, vol. 29, $\mathrm{N}^{\circ}$ 2, pp. 35-41.

CORDELL, V. V.

1991 «Competitive Context and Price as Moderators of Country of Origin Preferences». En: Journal of the Academy of Marketing Science, vol. 19, $N^{\circ} 2$, pp. 123-128.

DARLING, J. R. y V. R. WOOD

1990 «A Longitudinal Study Comparing Perceptions of US and Japanese Consumer Products in a Third/Neutral Country: Finland 1975 to 1985». En: Journal of International Business Studies, vol. 21, №3, pp. 427-450.

DINNIE, K.

2008 Nation Branding; Concepts, Issues, Practice. $1^{\text {a }}$ edic. Reino Unido: ButterworthHeinemann.

DOUGLAS, S.P. y S. CRAIG

1983 International Marketing Research. Englewood Cliffs, NJ: Prentice-Hall.

DOYLE, P.

1992 «Branding». En: BAKER, M. J. (ed.). The Marketing Book. $2^{\text {a }}$ edic. Reino Unido: Butterworth-Heinemann.

ERICKSON, G.M., JOHANSSON, J.K. y CHAO, P. 1984 Image variables in multi-attitude product evaluations: country-of-origin effects. Journal of Consumer Research, Vol. 11, September, pp. 694-9.

GAEDEKE, R.

1973 «Consumer Attitudes Toward Products 'Made In' Developing Countries». En: Journal of Retailing, vol. 49, №2, pp. 13-24.

HAN, C. M.

1989 "Country Image: Halo or Summary Construct?». En: Journal of Marketing Research, vol. 26, № 2, pp. 222-229. 
HAN, C. M. y V. TERPSTA

1988 «Country of Origin Effects for Uni-National y Bi-National Products». En: Journal of International Business Studies, vol. 19, $\mathrm{N}^{\circ}$ 2, pp. 235-55

HIGSON, A.

1997 "Nationality and the media». En: BRIGGS, A. y P. COBLEY (eds.). The Media: An Introduction. Londres: Addison Wesley Longman, pp. 354-364.

HIRSHMAN, E. C.

1981 «American Jewish Ethnicity; Its Relationship to Some Selected Aspects of Consumer Behavior». En: Journal of Marketing, vol. 45, $N^{\circ}$ 3, pp. 102-110.

JOHANSSON, J. K.; DOUGLAS, S. P. e I. NONAKA 1985 «Assessing the Impact of Country of Origin on Products Evaluations: A New Methodological Perspective». En: Journal of Marketing Research, vol. 22, $N^{\circ} 4$, pp. 388396.

KELLER, K. L.

2003 Strategic Brand Management: Building, Measuring, and Managing Brand Equity. $2^{\mathrm{a}}$ edic. Estados Unidos: Prentice Hall.

KLEIN, G., R. ETTENSON y M. D. MORRIS

1998 "The Animosity Model of Foreign Product Purchase: An Empirical Test in the People's Republic of China». En: Journal of Marketing, vol. 62, $N^{\circ} 1$, pp. 89-100.

KLEPPE, I. A.; N. M. IVERSEN E I. G. STENSAKER 2002 "Country Images in Marketing Strategies: Conceptual Issues and an Empirical Asian Illustration». En: Journal of Brand Management, vol. 10, $N^{\circ} 1, p p .61-74$.

KOTLER, P. y D. GERTNER

2002 «Country as Brand, Product, and Beyond: A Place Marketing and Brand Management Perspective». En: Journal of Brand Management, vol. 9, $\mathrm{N}^{\circ} 4$, pp. 249-261.
KOTLER, P. y KELLER, K. L.

2006 Marketing Management. 12 ${ }^{\mathrm{a}}$ edic. Pearson Prentice Hall.

KOTLER, P.; C. ASPLUND, L., REIN y D. H. HAIDER 1999 «Marketing Places Europe: Attracting Investment, Industry and Tourism to European Cities, Communities, States and Nations». En: Financial Times, p. 160.

KOTLER, P.; D. H. HAIDER y L., REIN

1993 Marketing Places: Attracting Investment, Industry and Tourism to Cities, States and Nations

LAWRENCE, C.; N. .E. MARR y G. P. PRENDERGAST 1992 "Country of Origin Stereotyping; A Case Study in the New Zealand Motor Vehicle Industry». En: Asia Pacific International Journal of Marketing, vol. $4 \mathrm{~N}^{\circ} 1$, pp. 37-51.

LECLERC, F.; Schmidt, B. H. y L. DUBÉ

1994 «Foreign Branding and Its Effects on Product Perceptions and Attitudes». En: Journal of Marketing Research, vol. 31, № 2, pp. 263270.

MORELLO, G.

1984 "The "Made In» Issue - A Comparative Research on the Image of Domestic and Foreign Products», en European Research, vol. 12, pp.5-21.

NAGASHIMA, A.

1970 «A Comparison of Japanese and US Attitudes toward Foreign Products». En: Journal of Marketing, vol. 34, $\mathrm{N}^{\circ} 1$, pp. 68-74.

OKECHUKU, $\mathrm{CH}$.

1994 «The Importance of Product Country of Origin: A Conjoint Analysis of the United States, Canada, Germany, and the Netherlands». En: European Journal of Marketing, vol. 28, N ${ }^{\circ}$ 4, pp. 5-19.

OLINS, W.

1999 Trading Identities: Why Countries and Companies are Taking each Others Roles. Londres: The Foreign Policy Centre. 
OLSON, J.C. y JACOBY, J.

1972 Cue utilisation in quality perception process en Venkatesan, M. (Ed.), Advance in Consumer Research, Third Annual Conference of the Association for Consumer Research, pp. 167-79.

\section{O'SHAUGHNESSY, J. y N. JACKSON}

2000 "Treating the Nation as a Brand: Some Neglected Issues». En: Journal of Macromarketing, vol. 20, № 1, pp. 56-64.

PAPADOPOULOS, N. G; L. A. HESLOP

2002 «Country Equity and Country Branding: Problems and Prospects». En: Journal of Brand Management, vol. 9, N 4/5, pp. 294314.

1987 Does Country-of-Origin Matter? Some Findings from a Cross-Cultural Study of Consumer Views about Foreign Products. Working Paper 87-104. Cambridge, MA: Marketing Science Institute.

PETERSON, R. A. y A. J.P. JOLIBERT

1995 "A Meta-Analysis of Country-Of-Origin Effects». En: Journal of International Business Studies, vol. 26, N 4, pp. 883-900.

PORTER, M.

1998 The Competitive Advantage of Nations. Londres: Palgrave.

RIEZEBOS, R.

2003 Brand Management: A Theorical and Practical Approach. Londres: FT Prentice Hall.
SAMIEE, S.

1994 "Customer Evaluation of Products in a Global Market». En: Journal of International Business Studies, vol. 25, №3, pp.579-604.

SCHOOLER, R.D.

1965 Product bias in the central American common market, "Journal of Marketing Research, November, pp. 394-7.

SMITH, A. D.

1991 National Identity. Londres: Penguin Books.

TEMPORAL, P.

2002 Advanced Brand Management: From Vision to Valuation. Singapur: John Wiley y Sons.

TUCK, J.

2003 «The Men in White». En: International Review for the Sociology of Sport, vol. 38, $N^{\circ} 2, p p$. 177-199.

USUNIER, J.C.

2006 Relevance in business research: the case of country-of-origin research in marketing. En: European Management Review. Houndmills: Spring 2006. Vol. 3, Iss. 1; p. 60

Vanossi, P.

2006 "Country as Brand: Nation Branding», disponible en h.ttp://www.affisch.org/weblog/archives/00000187.html 